\title{
Developing Finite Element Methods for Simulating Transformation Optics Devices with Metamaterials
}

\author{
Wei Yang ${ }^{1}$, Jichun $\mathrm{Li}^{2}{ }^{2}$, Yungqing Huang ${ }^{1}$ and Bin $\mathrm{He}^{1}$ \\ ${ }^{1}$ Hunan Key Laboratory for Computation and Simulation in Science and \\ Engineering, Xiangtan University, Xiangtan 411105, P.R. China. \\ 2 Department of Mathematical Sciences, University of Nevada Las Vegas, Las Vegas, \\ Nevada 89154-4020, USA.
}

Received 26 October 2017; Accepted (in revised version) 15 December 2017

\begin{abstract}
In this paper, we first develop the mathematical modeling equations for wave propagation in several transformation optics devices, including electromagnetic concentrator, rotator and splitter. Then we propose the corresponding finite element time-domain methods for simulating wave propagation in these transformation optics devices. We implement the proposed algorithms and our numerical results demonstrate the effectiveness of our modeling equations. To our best knowledge, this is the first work on time-domain finite element simulation carried out for the electromagnetic concentrator, rotator and splitter.
\end{abstract}

AMS subject classifications: 78M10, 65N30, 65F10, 78-08

Key words: Maxwell's equations, finite element time-domain methods, edge elements, transformation optics.

\section{Introduction}

Due to its many interesting potential applications, electromagnetic (EM) metamaterials has been one of the hot research topics since year 2000 as evidenced by many recently published papers (e.g., $[1,13,21,26,32,44]$ ) and monographs on metamaterials (e.g., $[22,29,35,37])$. Metamaterials and transformation optics (TO) offer some new techniques to tame the electromagnetic fields to achieve the desired applications in engineering and materials sciences [8,31]. In 2006, Pendry et al. [32] first presented the blueprints for realizing the EM cloak with metamaterials by using the TO theory. Since then, TO has attracted a great deal of attention $[8,10,36,37]$. TO can be applied to design many

*Corresponding author. Email addresses: yangweixtu@126.com (W. Yang), jichun. li@unlv.edu (J. Li), huangyq@xtu.edu.cn (Y. Huang), Hebinxtu@126.com (B. He) 
interesting metamaterial devices, such as cloaking device, concentrator, rotator, and splitter, etc. In 2008, Jiang et al. [20] designed the arbitrarily shaped concentrators based on the conformally optical transformation. In the same time, Chen and Chan [9] realized the EM rotator and splitter by a layered structure of thin alternating layers of metal and dielectrics based on the TO theory.

In order to understand the electromagnetic wave behavior in various media, we need to solve the famous Maxwell's equations:

$$
\begin{array}{ll}
\text { Ampere's law: } & \frac{\partial \boldsymbol{D}}{\partial t}=\nabla \times \boldsymbol{H}-\boldsymbol{J}_{s^{\prime}} \\
\text { Faraday's law: } & \frac{\partial \boldsymbol{B}}{\partial t}=-\nabla \times \boldsymbol{E}, \\
\text { Gauss's laws: } & \nabla \cdot \boldsymbol{D}=\rho, \quad \nabla \cdot \boldsymbol{B}=0,
\end{array}
$$

where $E, H, D, B$ denote the electric field, magnetic field, electric flux density, magnetic flux density, respectively, and $J_{s}, \rho$ denote the electric current and electric charge density, respectively. To make the Maxwell's equations well-posed, we often need to couple the Maxwell's equations with the constitutive equations for complex media:

$$
\boldsymbol{D}=\epsilon_{0} \epsilon \boldsymbol{E}, \quad \boldsymbol{B}=\mu_{0} \mu \boldsymbol{H},
$$

where $\epsilon_{0}$ and $\mu_{0}$ are the permittivity and permeability of vacuum, and $\epsilon$ and $\mu$ are the relative permittivity and permeability of the specific media.

There exist many excellent works on the analysis and applications of finite element method (FEM) for solving the Maxwell's equations in various media, such as in the free space (e.g., papers $[3,4,6,7,11,16,17,43]$, books $[12,22,30]$ and references therein); in general dispersive media (e.g., [2,19,28,34]); in negative index metamaterials [18,38,39]; and in cloaking metamaterials [23-25,41]. In recent years, we developed some mathematical models and time-domain FEMs for simulating the invisibility cloaks [23-25]. Continuing our previous efforts, here we develop some time-domain mathematical models and corresponding FEMs for simulating several transformation optics devices, including the EM concentrator $[20,33,36,41]$, rotator and splitter $[9,10]$. Transformation optics has become a fantastic tool to design these devices with novel functionalities. Numerical simulation plays a very important role in helping engineers and physicists demonstrating those novel functionalities they expect and then constructing the real physical devices by using the material parameters tested in the simulation. However, except [41] with self developed spectral-element solver to simulate the electromagnetic concentrators and rotators, almost all simulations of EM concentrator, rotator and splitter are performed in the frequency domain by using COMSOL, a popular commercial software in metamaterial community. As broadband devices are proposed and realized (e.g., broadband ground-plane cloak [27], broadband light absorber [31] and broadband rotator [10]), the time-domain simulation becomes more important and needed. To the best of our knowledge, developing these mathematical models and solving them by the time-domain FEMs is original 
and there is no existing work on this. The time-domain simulation is more interesting since the simulation can demonstrate how the incident wave interacts with the materials as it propagates, instead of just showing the steady state results obtained from frequency domain simulation. But the time-domain simulation is more challenging due to the complexity of the governing equations (with more unknowns and as a system of coupled differential equations) compared to just one governing equation in the frequency-domain modeling, and takes much longer computational time than the frequency-domain simulation.

The rest of the paper is organized as follows. In Section 2, we develop the timedomain mathematical governing equations for the TO devices such as the EM wave concentrator, rotator and splitter. In Section 3, we propose the fully discrete finite element schemes for solving our models. Then in Section 4, some interesting numerical results are presented to justify our model's effectiveness for describing the interesting wave propagation phenomena in these TO devices. Finally, we conclude our paper in Section 5.

\section{Mathematical models of three transformation optics devices}

In this section, we develop the time-domain mathematical model of some transformation optics (TO) devices, including electromagnetic concentrator, rotator and splitter. Firstly, we introduce how to obtain the physical parameters of these devices by mathematical transformation, then the dispersion properties of the TO metamaterials are clearly analyzed, finally we obtain the mathematical model by the mapping lossless Drude model to the dispersive physical parameters. The mathematical principle of these TO devices is based on the form invariant property of the Maxwell's equations.

Let us denote $\boldsymbol{E}(\boldsymbol{x})=\left(E_{1}, E_{2}\right)^{\prime}$ and $H(\boldsymbol{x})$ for the electric and magnetic fields, the $2 \times 2$ tensor $\epsilon$ and the scalar $\mu$ for the permittivity and permeability of the underlying material, respectively, and $2 \mathrm{D}$ curls

$$
\nabla \times \boldsymbol{E}=\frac{\partial E_{2}}{\partial x}-\frac{\partial E_{1}}{\partial y}, \quad \nabla \times H=\left(\frac{\partial H}{\partial y},-\frac{\partial H}{\partial x}\right)^{\prime} .
$$

Theorem 2.1 ([22]). Under a coordinate transformation $x^{\prime}=x^{\prime}(x)$, the $2 D$ time-harmonic Maxwell's equations

$$
\nabla \times \boldsymbol{E}(\boldsymbol{x})+j \omega \mu H(\boldsymbol{x})=0, \quad \nabla \times H(\boldsymbol{x})-j \omega \epsilon \boldsymbol{E}(\boldsymbol{x})=0,
$$

keep the same form in the transformed coordinate system:

$$
\nabla^{\prime} \times \boldsymbol{E}^{\prime}\left(\boldsymbol{x}^{\prime}\right)+j \omega \mu^{\prime} H^{\prime}(\boldsymbol{x})=0, \quad \nabla^{\prime} \times H^{\prime}\left(\boldsymbol{x}^{\prime}\right)-j \omega \epsilon^{\prime} \boldsymbol{E}^{\prime}(\boldsymbol{x})=0,
$$

where $\omega$ is the wave frequency, all variables in the old and new coordinate systems are related by

$$
\boldsymbol{E}^{\prime}\left(\boldsymbol{x}^{\prime}\right)=J^{-T} \boldsymbol{E}(\boldsymbol{x}), \quad H^{\prime}\left(\boldsymbol{x}^{\prime}\right)=H(\boldsymbol{x}), \quad \epsilon^{\prime}=\frac{J \epsilon J^{T}}{\operatorname{det}(J)}, \quad \mu^{\prime}=\frac{\mu(\boldsymbol{x})}{\operatorname{det}(J)},
$$

where $J=\left(\partial x_{i}^{\prime} / \partial x_{j}\right)$ denotes the Jacobian transformation matrix. 

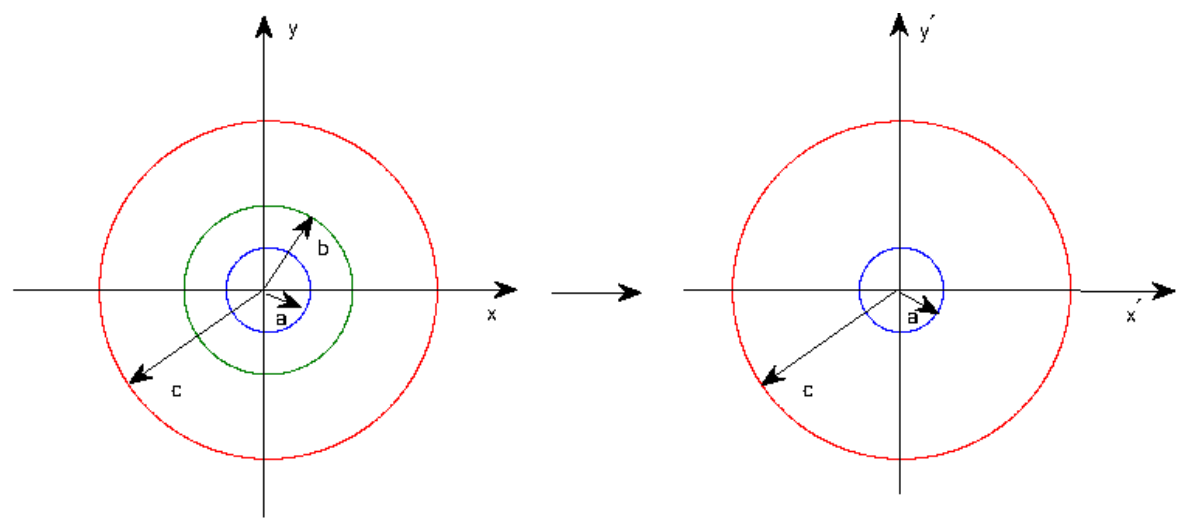

Figure 1: Coordinate transformation for cylindrical concentrator: (left) the original space; (right) the transformed space.

\subsection{Electromagnetic concentrator}

For the cylindrical concentrator, the ideal material parameters in the polar coordinate system can be derived from the coordinate transformation [33]:

$$
\begin{aligned}
& r^{\prime}= \begin{cases}\frac{a}{b} r, & 0 \leq r \leq b, \\
\frac{c-a}{c-b} r-\frac{b-a}{c-b} c, & b \leq r \leq c,\end{cases} \\
& \theta^{\prime}=\theta, \quad 0 \leq \theta \leq 2 \pi,
\end{aligned}
$$

which compresses the circle $r \leq b$ into a smaller circle $r^{\prime} \leq a$, and stretches the annulus $b \leq r \leq c$ to $a \leq r^{\prime} \leq c$. Because the stretch and compression are mutually compensated, which results in the consistency of the original space and the transformed space. The function of this transformation is to concentrate the energy in the inner area $r^{\prime} \leq a$.

In Cartesian coordinate system, the transformation (2.4) can be written as

$$
\begin{aligned}
& \left\{\begin{array}{l}
x^{\prime}=r^{\prime} \cos \theta^{\prime}=\frac{a}{b} x, \\
y^{\prime}=r^{\prime} \sin \theta^{\prime}=\frac{a}{b} y, \quad 0 \leq r^{\prime} \leq a,
\end{array}\right. \\
& \left\{\begin{array}{l}
x^{\prime}=r^{\prime} \cos \theta^{\prime}=\left(\frac{c-a}{c-b} r-\frac{b-a}{c-b} c\right) \cos \theta^{\prime}=\frac{c-a}{c-b} x-\frac{c(b-a)}{c-b} \cdot \frac{x}{r}, \quad a \leq r^{\prime} \leq c, \\
y^{\prime}=r^{\prime} \sin \theta^{\prime}=\left(\frac{c-a}{c-b} r-\frac{b-a}{c-b} c\right) \sin \theta^{\prime}=\frac{c-a}{c-b} y-\frac{c(b-a)}{c-b} \cdot \frac{y}{r},
\end{array}\right.
\end{aligned}
$$

from which we can derive the Jacobian transformation matrix:

$$
J=\left(\begin{array}{ll}
\frac{\partial x^{\prime}}{\partial x} & \frac{\partial x^{\prime}}{\partial y} \\
\frac{\partial y^{\prime}}{\partial x} & \frac{\partial y^{\prime}}{\partial y}
\end{array}\right)=\left\{\begin{array}{cc}
\left(\begin{array}{cc}
\frac{a}{b} & 0 \\
0 & \frac{a}{b}
\end{array}\right), & 0 \leq r^{\prime} \leq a, \\
\left(\begin{array}{cc}
\frac{c-a}{c-b}-\frac{c(b-a) \sin ^{2} \theta^{\prime}}{(c-b) r} & \frac{c(b-a) \sin \theta^{\prime} \cos \theta^{\prime}}{(c-b) r} \\
* & \frac{c-a}{c-b}-\frac{c(b-a) \cos ^{2} \theta^{\prime}}{(c-b) r}
\end{array}\right), & a \leq r^{\prime} \leq c .
\end{array}\right.
$$


Here and below we denote " $*$ " for the symmetric part.

It is easy to check that

$$
\operatorname{det}(J)= \begin{cases}\left(\frac{a}{b}\right)^{2}, & 0 \leq r^{\prime} \leq a, \\ \frac{(c-a) r^{\prime}}{(c-b) r}, & a \leq r^{\prime} \leq c .\end{cases}
$$

Then from Theorem 2.1, we can obtain the relative permittivity and permeability for the concentrator:

$$
\begin{aligned}
& \epsilon^{\prime}=\left\{\begin{array}{cc}
\left(\begin{array}{ll}
1 & 0 \\
0 & 1
\end{array}\right), & 0 \leq r^{\prime} \leq a, \\
\left(\begin{array}{ll}
\frac{1}{K_{2}}-\frac{K_{1}}{r}\left(\frac{2}{\sqrt{K_{2}}}-\frac{K_{1}}{r}\right) \sin ^{2} \theta^{\prime} \\
K_{2}-\frac{K_{1} \sqrt{K_{2}}}{r} & \frac{\frac{K_{1}}{r}\left(\frac{2}{\sqrt{K_{2}}}-\frac{K_{1}}{r}\right) \sin \theta^{\prime} \cos \theta^{\prime}}{K_{2}-\frac{K_{1} \sqrt{K_{2}}}{r}} \\
* & \frac{\frac{1}{K_{2}}-\frac{K_{1}}{r}\left(\frac{2}{\sqrt{K_{2}}}-\frac{K_{1}}{r}\right) \cos ^{2} \theta^{\prime}}{K_{2}-\frac{K_{1} \sqrt{K_{2}}}{r}}
\end{array}\right), a \leq r^{\prime} \leq c,
\end{array}\right. \\
& \mu^{\prime}= \begin{cases}b^{2} / a^{2}, & 0 \leq r^{\prime} \leq a, \\
K_{2}\left(r^{\prime}+K_{1}\right) / r^{\prime}, & a \leq r^{\prime} \leq c,\end{cases}
\end{aligned}
$$

where we denote constants

$$
K_{1}=\frac{(b-a) c}{c-b}, \quad K_{2}=\left(\frac{c-b}{c-a}\right)^{2} .
$$

For the real symmetric matrix $\epsilon^{\prime}$, we can diagonalize of the permittivity matrix of the concentrator [24, 42]:

$$
\begin{aligned}
\epsilon^{\prime}\left(r^{\prime}\right) & =\left(\begin{array}{cc}
\cos \theta & -\sin \theta \\
\sin \theta & \cos \theta
\end{array}\right)\left(\begin{array}{cc}
\epsilon_{r} & 0 \\
0 & \epsilon_{\theta}
\end{array}\right)\left(\begin{array}{cc}
\cos \theta & \sin \theta \\
-\sin \theta & \cos \theta
\end{array}\right) \\
& =\left(\begin{array}{cc}
\epsilon_{r} \cos ^{2} \theta+\epsilon_{\theta} \sin ^{2} \theta & \left(\epsilon_{r}-\epsilon_{\theta}\right) \sin \theta \cos \theta \\
* & \epsilon_{r} \sin ^{2} \theta+\epsilon_{\theta} \cos ^{2} \theta
\end{array}\right), \quad a \leq r^{\prime} \leq c,
\end{aligned}
$$

where

$$
\epsilon_{r}=\frac{r^{\prime}+K_{1}}{r^{\prime}}, \quad \epsilon_{\theta}=\frac{r^{\prime}}{r^{\prime}+K_{1}} .
$$

From (2.9), we can see that

$$
\frac{a+K_{1}}{a} \geq \epsilon_{r} \geq \frac{c+K_{1}}{c}>1, \quad \frac{a}{a+K_{1}} \leq \epsilon_{\theta} \leq \frac{c}{c+K_{1}}<1,
$$

i.e., the physical parameters of concentrator are bounded below and above, which are different from the cloaking metamaterials that are unbounded [22]. Note that $\epsilon_{\theta}<1$, 
which is nonphysical and is often mapped by a dispersive medium model in numerical simulation [15]. Here we adopt the lossless Drude model:

$$
\epsilon_{\theta}=\epsilon_{\max }-\frac{\omega_{e}^{2}}{\omega^{2}}
$$

where $\epsilon_{\max }=c /\left(c+K_{1}\right)$ is the maximum value of $\epsilon_{\theta}$, and $\omega>0$ and $\omega_{e}>0$ are the source wave and plasma frequencies, respectively. Following our previous work [24], substituting (2.8) into the constitutive equation $\boldsymbol{D}=\epsilon_{0} \epsilon^{\prime} \boldsymbol{E}$, we obtain

$$
\epsilon_{0} \epsilon_{r} \epsilon_{\theta} E=\left(\begin{array}{cc}
\epsilon_{r} \sin ^{2} \theta+\epsilon_{\theta} \cos ^{2} \theta & \left(\epsilon_{r}-\epsilon_{\theta}\right) \sin \theta \cos \theta \\
* & \epsilon_{r} \cos ^{2} \theta+\epsilon_{\theta} \sin ^{2} \theta
\end{array}\right) D .
$$

Then substituting (2.10) into (2.11), we have

$$
\epsilon_{0} \epsilon_{r}\left(\epsilon_{\max }-\frac{\omega_{e}^{2}}{\omega^{2}}\right) E=\left(\begin{array}{cc}
\epsilon_{r} \sin ^{2} \theta+\left(\epsilon_{\max }-\frac{\omega_{e}^{2}}{\omega^{2}}\right) \cos ^{2} \theta & \left(\epsilon_{r}-\left(\epsilon_{\max }-\frac{\omega_{e}^{2}}{\omega^{2}}\right)\right) \sin \theta \cos \theta \\
* & \epsilon_{r} \cos ^{2} \theta+\left(\epsilon_{\max }-\frac{\omega_{e}^{2}}{\omega^{2}}\right) \sin ^{2} \theta
\end{array}\right) \boldsymbol{D} .
$$

Changing the above equation into time domain by using rules

$$
j \omega \rightarrow \frac{\partial}{\partial t^{\prime}}, \omega^{2} \rightarrow-\frac{\partial^{2}}{\partial t^{2}}
$$

we obtain

$$
\epsilon_{0} \epsilon_{r}\left(\epsilon_{\max } \boldsymbol{E}_{t t}+\omega_{e}^{2} \boldsymbol{E}\right)=M_{c a} \boldsymbol{D}_{t t}+M_{c b} \boldsymbol{D},
$$

where

$$
M_{c a}=\left[\begin{array}{cc}
\epsilon_{r} \sin ^{2} \phi+\epsilon_{\max } \cos ^{2} \phi & \left(\epsilon_{r}-\epsilon_{\max }\right) \sin \phi \cos \phi \\
* & \epsilon_{r} \cos ^{2} \phi+\epsilon_{\max } \sin ^{2} \phi
\end{array}\right], \quad M_{c b}=\omega_{e}^{2}\left[\begin{array}{cc}
\cos ^{2} \phi & \sin \phi \cos \phi \\
* & \sin ^{2} \phi
\end{array}\right] .
$$

Lemma 2.1. The relative permeability $\mu^{\prime}\left(r^{\prime}\right)=K_{2} \frac{r^{\prime}+K_{1}}{r^{\prime}}$ of EM concentrator is smaller than one at some points in the domain $r^{\prime} \in[a, c]$.

Proof. Let $r_{0}^{\prime}=c-\delta$ for some $\delta \in(0, c-a)$. To guarantee $\mu^{\prime}\left(r_{0}^{\prime}\right)<1$, we need make sure that there exists some parameter $\delta$ such that

$$
\mu^{\prime}\left(r_{0}^{\prime}\right)=K_{2}\left(1+\frac{K_{1}}{c-\delta}\right)<1 .
$$

By simple algebra, we obtain

$$
\delta<\frac{c-K_{2} c-K_{1} K_{2}}{1-K_{2}}=\frac{c(c-a)}{2 c-a-b},
$$

which means that such $\delta$ indeed exists. For example, taking

$$
\delta=\frac{c-a}{2}<\frac{c(c-a)}{2 c-a-b}
$$


leads to

$$
r_{0}^{\prime}=\frac{a+c}{2} \in(a, c), \quad \text { and } \quad \mu^{\prime}\left(r_{0}^{\prime}\right)=\frac{(c-b)(c+b)}{(c-a)(c+a)}<1 .
$$

This completes our proof.

Lemma 2.1 shows that the permeability $\mu^{\prime}\left(r^{\prime}\right)<1$ at some points and hence it needs to be mapped by a dispersive medium model. We choose the following Drude model:

$$
\mu^{\prime}\left(x^{\prime}\right)=\mu_{\max }-\frac{\omega_{m}^{2}}{\omega^{2}}
$$

where

$$
\mu_{\max }=\frac{K_{2}\left(a+K_{1}\right)}{a}=\frac{b(c-b)}{a(c-a)}
$$

is the maximum value of $\mu^{\prime}\left(\boldsymbol{x}^{\prime}\right)$, and $\omega_{m}>0$ is magnetic plasma frequency, respectively. Substituting (2.15) into the constitutive equation $B=\mu_{0} \mu^{\prime} H$, and using rules (2.12), we have

$$
\mu_{0}\left(\mu_{\max } H_{t t}+\omega_{m}^{2} H\right)=B_{t t} .
$$

Coupling Eqs. (2.13) and (2.16) with Faraday's and Ampere's law, we can obtain the timedomain governing equations for electromagnetic concentrator:

$$
\begin{aligned}
& \boldsymbol{D}_{t}=\nabla \times H, \\
& \epsilon_{0} \epsilon_{r}\left(\epsilon_{\max } \boldsymbol{E}_{t t}+\omega_{e}^{2} \boldsymbol{E}\right)=M_{c a} \boldsymbol{D}_{t t}+M_{c b} \boldsymbol{D}, \\
& B_{t}=-\nabla \times \boldsymbol{E}, \\
& \mu_{0}\left(\mu_{\max } H_{t t}+\omega_{m}^{2} H\right)=B_{t t} .
\end{aligned}
$$

\subsection{Electromagnetic rotator}

In this subsection, we consider a 2D cylindrical EM rotator. This device is formed by two regions: the shell region $\Omega_{2}=\left\{(r, \theta): R_{1} \leq r \leq R_{2}, 0 \leq \theta \leq 2 \pi\right\}$ is used to change the direction of wave propagation, and the core region $\Omega_{1}=\left\{(r, \theta): 0 \leq r \leq R_{1}, 0 \leq \theta \leq 2 \pi\right\}$ is used to rotate the incoming EM wave by an angle $\theta_{0}$ when it leaves this region (cf., Fig. 2). For the cylindrical EM rotator, the exact material parameters in the polar coordinate system can be obtained by the transformation [9]:

$$
\begin{aligned}
& r^{\prime}=r, \quad 0 \leq r \leq R_{2}, \\
& \theta^{\prime}= \begin{cases}\theta+\theta_{0}, & 0 \leq r \leq R_{1}, \\
\theta+\xi, & R_{1} \leq r \leq R_{2},\end{cases}
\end{aligned}
$$

where $\xi=\theta_{0}\left(R_{2}-r\right) /\left(R_{2}-R_{1}\right)$. It is easy to see that the transformation (2.18) rotates the incoming wave by an angle $\theta_{0}$ in the core region $\Omega_{1}$, and then gradually reduces the rotated angle $\theta_{0}$ to 0 in the shell region. 


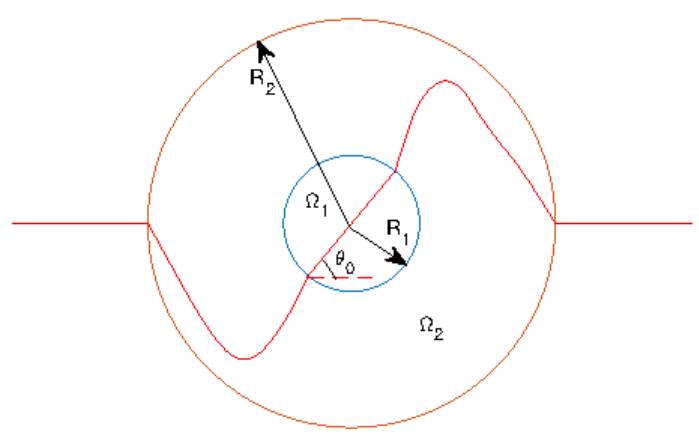

Figure 2: Coordinate transformation of a cylindrical EM rotator.

In the Cartesian coordinate system, the transformation (2.18) can be written as

$$
\begin{aligned}
& \left\{\begin{array}{l}
x^{\prime}=r^{\prime} \cos \theta^{\prime}=r^{\prime} \cos \left(\theta+\theta_{0}\right)=x \cos \theta_{0}-y \sin \theta_{0}, \\
y^{\prime}=r^{\prime} \sin \theta^{\prime}=r^{\prime} \sin \left(\theta+\theta_{0}\right)=x \sin \theta_{0}+y \cos \theta_{0},
\end{array} \quad 0 \leq r^{\prime} \leq R_{1},\right. \\
& \left\{\begin{array}{l}
x^{\prime}=r^{\prime} \cos \theta^{\prime}=r \cos (\theta+\xi)=x \cos \xi-y \sin \xi, \\
y^{\prime}=r^{\prime} \sin \theta^{\prime}=r \sin (\theta+\xi)=x \sin \xi+y \cos \xi,
\end{array} \quad R_{1} \leq r^{\prime} \leq R_{2},\right.
\end{aligned}
$$

from which we can obtain the Jacobian transformation matrix:

$$
J=\left(\begin{array}{ll}
\frac{\partial x^{\prime}}{\partial x} & \frac{\partial x^{\prime}}{\partial y} \\
\frac{\partial y^{\prime}}{\partial x} & \frac{\partial y^{\prime}}{\partial y}
\end{array}\right)= \begin{cases}\left(\begin{array}{cc}
\cos \theta_{0} & -\sin \theta_{0} \\
\sin \theta_{0} & \cos \theta_{0}
\end{array}\right), & 0 \leq r^{\prime} \leq R_{1} \\
\left(\begin{array}{cc}
\cos \xi-\frac{\partial \xi}{\partial x} y^{\prime} & -\sin \xi-\frac{\partial \xi}{\partial y} y^{\prime} \\
\sin \xi+\frac{\partial \xi}{\partial x} x^{\prime} & \cos \xi+\frac{\partial \xi}{\partial y} x^{\prime}
\end{array}\right), & R_{1} \leq r^{\prime} \leq R_{2}\end{cases}
$$

where

$$
\frac{\partial \xi}{\partial x}=\frac{-\theta_{0}}{R_{2}-R_{1}} \cdot \frac{\partial r}{\partial x}=-m \frac{x}{r^{2}}, \quad \frac{\partial \xi}{\partial y}=\frac{-\theta_{0}}{R_{2}-R_{1}} \cdot \frac{\partial r}{\partial y}=-m \frac{y}{r^{2}}, \quad m=\frac{\theta_{0} r^{\prime}}{R_{2}-R_{1}}
$$

It is easy to check that $\operatorname{det}(J)=1$ for all $0 \leq r^{\prime} \leq R_{2}$. By the TO theory, the relative permittivity and permeability for the EM rotator can be obtained as follows:

$$
\begin{aligned}
& \epsilon^{\prime}\left(x^{\prime}\right)= \begin{cases}\left(\begin{array}{ll}
1 & 0 \\
0 & 1
\end{array}\right), & 0 \leq r^{\prime} \leq R_{1} \\
\left(\begin{array}{ll}
a\left(x^{\prime}\right) & b\left(x^{\prime}\right) \\
b\left(x^{\prime}\right) & c\left(x^{\prime}\right)
\end{array}\right), & R_{1} \leq r^{\prime} \leq R_{2}\end{cases} \\
& \mu^{\prime}\left(x^{\prime}\right)=1, \quad 0 \leq r^{\prime} \leq R_{2},
\end{aligned}
$$


where

$$
\begin{aligned}
& a\left(x^{\prime}\right)=1+2 m \frac{x^{\prime} y^{\prime}}{r^{2}}+m^{2} \frac{\left(y^{\prime}\right)^{2}}{r^{2}}, \\
& b\left(x^{\prime}\right)=-m\left(\frac{\left(x^{\prime}\right)^{2}}{r^{2}}-\frac{\left(y^{\prime}\right)^{2}}{r^{2}}\right)-m^{2} \frac{x^{\prime} y^{\prime}}{r^{2}}, \\
& c\left(x^{\prime}\right)=1+\frac{\left(x^{\prime}\right)^{2}}{r^{2}} m^{2}-2 m \frac{x^{\prime} y^{\prime}}{r^{2}} .
\end{aligned}
$$

Through simple algebra, we can obtain the eigenvalues of relative permittivity $\epsilon^{\prime}\left(\boldsymbol{x}^{\prime}\right)$ on the shell region $R_{1} \leq r^{\prime} \leq R_{2}$ :

$$
0<\lambda_{r 1}=\frac{2+m^{2}-\sqrt{\left(2+m^{2}\right)^{2}-4}}{2}<1, \quad \lambda_{r 2}=\frac{2+m^{2}+\sqrt{\left(2+m^{2}\right)^{2}-4}}{2}>1,
$$

and diagonalize the real symmetric matrix $\epsilon^{\prime}$ (cf. [25]):

$$
\epsilon^{\prime}\left(\boldsymbol{x}^{\prime}\right)=P\left[\begin{array}{cc}
\lambda_{r 1} & 0 \\
0 & \lambda_{r 2}
\end{array}\right] P^{T}, \quad P=\left[\begin{array}{cc}
p_{1} & p_{2} \\
-p_{2} & p_{1}
\end{array}\right]
$$

where

$$
p_{1}=\sqrt{\frac{\lambda_{r 2}-a\left(x^{\prime}\right)}{\lambda_{r 2}-\lambda_{r 1}}}, \quad p_{2}=\operatorname{sgn}\left(b\left(x^{\prime}\right)\right) \sqrt{\frac{a\left(x^{\prime}\right)-\lambda_{r 1}}{\lambda_{r 2}-\lambda_{r 1}}} .
$$

Here sgn represents the standard sign function.

Because the eigenvalue $\lambda_{r 1} \in(0,1)$, which is nonphysical and we map in by the lossless Drude dispersive medium model $\lambda_{r 1}=1-\omega_{r e}^{2} / \omega^{2}$, where $\omega_{r e}>0$ is the electric plasma frequency.

Following the similar derivations in Subsection 2.1, we can obtain the time-domain governing equations for the EM rotator in subdomain $\Omega_{2}$ :

$$
\begin{aligned}
& \boldsymbol{D}_{t}=\nabla \times H, \\
& \epsilon_{0} \lambda_{r 2}\left(\boldsymbol{E}_{t t}+\omega_{r e}^{2} \boldsymbol{E}\right)=M_{r a} \boldsymbol{D}_{t t}+M_{r b} \boldsymbol{D}, \\
& \mu_{0} H_{t}=-\nabla \times \boldsymbol{E},
\end{aligned}
$$

where the matrices $M_{r a}$ and $M_{r b}$ are given as

$$
M_{r a}=\left[\begin{array}{cc}
p_{1}^{2} \lambda_{r 2}+p_{2}^{2} & p_{1} p_{2}-p_{1} p_{2} \lambda_{r 2} \\
* & p_{1}^{2}+p_{2}^{2} \lambda_{r 2}
\end{array}\right], \quad M_{r b}=\omega_{r e}^{2}\left[\begin{array}{cc}
p_{2}^{2} & p_{1} p_{2} \\
* & p_{1}^{2}
\end{array}\right] .
$$

Because the relative permittivity and permeability in $\Omega_{1}$ are one, the governing equations in $\Omega_{1}$ are the standard Maxwell's equation in free space. 


\subsection{Electromagnetic splitter}

In this subsection, we consider the time-domain mathematical model of an EM splitter. The EM splitter is made of two parts: a lifting device and a moving down device. The lifting medium is designed by the following transformation [9] (cf. Fig. 3):

$$
\begin{aligned}
& x^{\prime}=x, y^{\prime}=y, \quad \text { for } x<x_{1}, \\
& x^{\prime}=x, y^{\prime}=y+k\left(x-x_{1}\right), \quad \text { for } x_{1} \leq x \leq x_{2}, \\
& x^{\prime}=x, y^{\prime}=y+k\left(x_{2}-x_{1}\right), \text { for } x>x_{2} \text {, }
\end{aligned}
$$

where the constant $k>0$ is called "moving parameter". In transformation (2.25), we can control the propagation direction of the incoming EM wave. The wave moves up $k\left(x_{2}-x_{1}\right)$ along the $y$ direction after the wave passes through the region $x_{1}<x<x_{2}$.
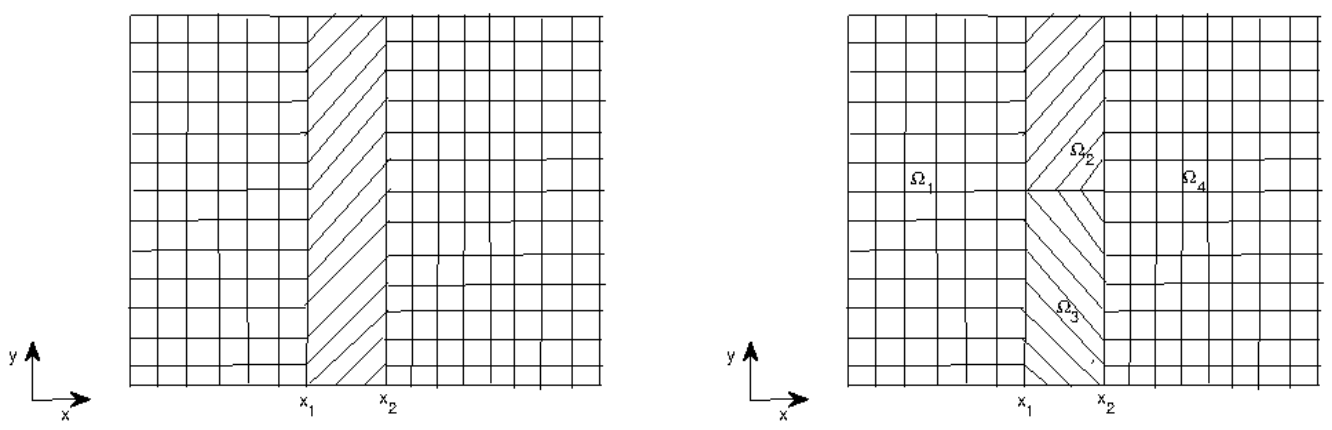

Figure 3: Coordinate transformation for the EM lifting device (left), and splitter (right).

By Theorem 2.1, we can obtain the relative permittivity and permeability:

$$
\begin{aligned}
& \epsilon^{\prime}\left(x^{\prime}\right)=\left[\begin{array}{cc}
1 & k \\
k & 1+k^{2}
\end{array}\right], \quad \mu^{\prime}\left(x^{\prime}\right)=1, \quad \text { for } x_{1} \leq x^{\prime} \leq x_{2} \\
& \epsilon^{\prime}\left(x^{\prime}\right)=\left[\begin{array}{ll}
1 & 0 \\
0 & 1
\end{array}\right], \quad \mu^{\prime}\left(x^{\prime}\right)=1, \text { for } x^{\prime} \leq x_{1}, \text { or } x^{\prime} \geq x_{2} .
\end{aligned}
$$

It is easy to obtain the eigenvalues of relative permittivity $\epsilon^{\prime}\left(x^{\prime}\right)$ in $\Omega_{2}$ as follows:

$$
0<\lambda_{s 1}=\frac{2+k^{2}-\sqrt{\left(2+k^{2}\right)^{2}-4}}{2}<1, \quad \lambda_{s 2}=\frac{2+k^{2}+\sqrt{\left(2+k^{2}\right)^{2}-4}}{2}>1 .
$$

Following similar steps to Subsection 2.2 and using the following Drude model for $\lambda_{s 1}$ :

$$
\lambda_{s 1}=1-\frac{\omega_{s e}^{2}}{\omega^{2}}
$$


we can obtain the time-domain governing equations for the EM lifting device in $\Omega_{2}$ :

$$
\begin{aligned}
& \boldsymbol{D}_{t}=\nabla \times H, \\
& \epsilon_{0} \lambda_{s 2}\left(\boldsymbol{E}_{t t}+\omega_{s e}^{2} \boldsymbol{E}\right)=M_{s a} \boldsymbol{D}_{t t}+M_{s b} \boldsymbol{D}, \\
& \mu_{0} H_{t}=-\nabla \times \boldsymbol{E},
\end{aligned}
$$

where

$$
M_{s a}=\left[\begin{array}{cc}
p_{s 1}^{2} \lambda_{s 2}+p_{2}^{2} & p_{s 1} p_{s 2}-p_{s 1} p_{s 2} \lambda_{s 2} \\
* & p_{s 1}^{2}+p_{s 2}^{2} \lambda_{s 2}
\end{array}\right], \quad M_{s b}=\omega_{s e}^{2}\left[\begin{array}{cc}
p_{s 2}^{2} & p_{s 1} p_{s 2} \\
* & p_{s 1}^{2}
\end{array}\right],
$$

with

$$
p_{s 1}=\sqrt{\frac{k+\sqrt{k^{2}+4}}{2 \sqrt{k^{2}+4}}}, \quad p_{s 2}=\sqrt{\frac{\sqrt{k^{2}+4}-k}{2 \sqrt{k^{2}+4}}} .
$$

The governing equations in $\Omega_{1}$ and $\Omega_{4}$ (cf. Fig. 3) are the standard Maxwell's equation in free space.

Choosing the moving parameter $k<0$ in the transformation (2.25), we obtain the moving down device. The governing equations for the moving down device are the same as the lifting device except

$$
p_{s 2}=-\sqrt{\frac{\sqrt{k^{2}+4}-k}{2 \sqrt{k^{2}+4}}} .
$$

We can obtain a wave splitter by coupling the lifting and moving down devices together (cf. Fig. 3).

\section{Time-domain finite element schemes for TO devices}

To design the finite element method for modelling TO devices, we partition the physical domain $\Omega$ by a family of regular meshes $\mathcal{T}_{h}$ with maximum mesh size $h$. To accommodate the complicated geometric domain, we use rectangles in the perfectly matched layer (PML) region, and triangles elsewhere.

For simplicity, we only implement the lowest-order Raviart-Thomas-Nédélec's mixed finite element spaces $U_{h}$ and $V_{h}$ given as follows: on any rectangular element $e \in \mathcal{T}_{h}$, we choose

$$
\begin{aligned}
& U_{h}=\left\{\psi_{h} \in L^{2}(\Omega):\left.\psi_{h}\right|_{e} \text { is a constant } \forall e \in \mathcal{T}_{h}\right\}, \\
& V_{h}=\left\{\phi_{h} \in H(\text { curl } ; \Omega):\left.\phi_{h}\right|_{e} \in Q_{0,1} \times Q_{1,0} \quad \forall e \in \mathcal{T}_{h}\right\},
\end{aligned}
$$

where $Q_{i, j}$ denotes polynomials whose degrees are less than or equal to $i$ and $j$ in variables $x$ and $y$, respectively; while on a triangular element, we choose

$$
\begin{aligned}
& U_{h}=\left\{\psi_{h} \in L^{2}(\Omega):\left.\psi_{h}\right|_{e} \text { is a constant } \forall e \in \mathcal{T}_{h}\right\}, \\
& V_{h}=\left\{\phi_{h} \in H(\operatorname{curl} ; \Omega):\left.\phi_{h}\right|_{e} \in \operatorname{span}\left\{\phi_{i} \nabla \phi_{j}-\phi_{j} \nabla \phi_{i}\right\}, i, j=1,2,3 \forall e \in \mathcal{T}_{h}\right\},
\end{aligned}
$$


where $\phi_{i}$ denotes the barycentric coordinates of element $e$. To impose the perfect conducting boundary condition $n \times E=0$, we introduce the subspace

$$
\boldsymbol{V}_{h}^{0}=\left\{\phi_{h} \in \boldsymbol{V}_{h}: \boldsymbol{n} \times \phi_{h}=0 \text {, on } \partial \Omega\right\},
$$

where $n$ is the unit normal vector on $\partial \Omega$.

To define a fully-discrete scheme, we divide the time interval $I=[0, T]$ into $N$ uniform subintervals $I_{i}=\left[t_{i-1}, t_{i}\right]$ by points $t_{i}=i \tau, i=0,1, \cdots, N$, where $\tau=T / N$. For time solutions $E^{n}$, we introduce backward difference operators:

$$
\delta_{\tau} \boldsymbol{E}^{n+\frac{1}{2}}=\frac{\boldsymbol{E}^{n+\frac{1}{2}}-\boldsymbol{E}^{n-\frac{1}{2}}}{\tau}, \quad \delta_{\tau}^{2} \boldsymbol{E}^{n+\frac{1}{2}}=\frac{\boldsymbol{E}^{n+\frac{1}{2}}-2 \boldsymbol{E}^{n-\frac{1}{2}}+\boldsymbol{E}^{n-\frac{3}{2}}}{\tau^{2}},
$$

and average operators:

$$
\overline{\boldsymbol{E}}^{n+\frac{1}{2}}=\frac{\boldsymbol{E}^{n+\frac{1}{2}}+2 \boldsymbol{E}^{n-\frac{1}{2}}+\boldsymbol{E}^{n-\frac{3}{2}}}{4}, \quad \widehat{\boldsymbol{E}}^{n+\frac{1}{2}}=\frac{\boldsymbol{E}^{n+\frac{1}{2}}+\boldsymbol{E}^{n-\frac{1}{2}}}{2} .
$$

With the above preparations, now we can construct our time-domain finite element schemes for solving those TO modeling equations.

EM concentrator: Given initial approximations $\boldsymbol{E}_{h}^{-\frac{3}{2}}, \boldsymbol{E}_{h}^{-\frac{1}{2}}, \boldsymbol{D}_{h}^{-\frac{3}{2}}, \boldsymbol{D}_{h}^{-\frac{1}{2}}, H_{h}^{0}$, for any $n \geq 0$, find $\boldsymbol{D}_{h}^{n+\frac{1}{2}} \in \boldsymbol{V}_{h}^{0}, \boldsymbol{E}_{h}^{n+\frac{1}{2}} \in \boldsymbol{V}_{h}^{0}, H_{h}^{n+1} \in U_{h}$ such that

$$
\begin{aligned}
& \left(\delta_{\tau} \boldsymbol{D}_{h}^{n+\frac{1}{2}}, v_{h}\right)=\left(H_{h}^{n}, \nabla \times v_{h}\right), \\
& \epsilon_{0} \epsilon_{\max }\left(\epsilon_{r} \delta_{\tau}^{2} \boldsymbol{E}_{h}^{n+\frac{1}{2}}, \phi_{h}\right)+\epsilon_{0}\left(\omega_{e}^{2} \epsilon_{r} M \overline{\boldsymbol{E}}_{h}^{n+\frac{1}{2}}, \phi_{h}\right)=\left(M_{c a} \delta_{\tau}^{2} \boldsymbol{D}_{h}^{n+\frac{1}{2}}, \phi_{h}\right)+\left(M_{c b} \overline{\boldsymbol{D}}_{h}^{n+\frac{1}{2}}, \phi_{h}\right), \\
& \mu_{0} \mu_{\max }\left(\delta_{\tau}^{2} H_{h}^{n+1}, \psi_{h}\right)+\mu_{0}\left(\omega_{m}^{2} \bar{H}_{h}^{n+1}, \psi_{h}\right)=-\left(\nabla \times \delta_{\tau} \widehat{\boldsymbol{E}}_{h}^{n+\frac{1}{2}}, \psi_{h}\right),
\end{aligned}
$$

hold true for any $v_{h} \in V_{h}^{0}, \phi_{h} \in V_{h}^{0}, \psi_{h} \in U_{h}$.

EM rotator: Given initial approximations $\boldsymbol{E}_{h}^{-\frac{3}{2}}, \boldsymbol{E}_{h}^{-\frac{1}{2}}, \boldsymbol{D}_{h}^{-\frac{3}{2}}, \boldsymbol{D}_{h}^{-\frac{1}{2}}, H_{h}^{0}$, for any $n \geq 0$, find $\boldsymbol{D}_{h}^{n+\frac{1}{2}} \in \boldsymbol{V}_{h}^{0}, \boldsymbol{E}_{h}^{n+\frac{1}{2}} \in \boldsymbol{V}_{h}^{0}, H_{h}^{n+1} \in U_{h}$ such that

$$
\begin{aligned}
& \left(\delta_{\tau} \boldsymbol{D}_{h}^{n+\frac{1}{2}}, v_{h}\right)=\left(H_{h}^{n}, \nabla \times v_{h}\right), \\
& \epsilon_{0}\left(\lambda_{r 2} \delta_{\tau}^{2} E_{h}^{n+\frac{1}{2}}, \phi_{h}\right)+\epsilon_{0}\left(\lambda_{r 2} \omega_{r e}^{2} \overline{\boldsymbol{E}}_{h}^{n+\frac{1}{2}}, \phi_{h}\right)=\left(M_{r a} \delta_{\tau}^{2} \boldsymbol{D}_{h}^{n+\frac{1}{2}}, \phi_{h}\right)+\left(M_{r b} \overline{\boldsymbol{D}}_{h}^{n+\frac{1}{2}}, \phi_{h}\right), \\
& \mu_{0}\left(\delta_{\tau} H_{h}^{n+1}, \psi_{h}\right)=-\left(\nabla \times \boldsymbol{E}_{h}^{n+\frac{1}{2}}, \psi_{h}\right),
\end{aligned}
$$

hold true for any $v_{h} \in V_{h}^{0}, \phi_{h} \in V_{h}^{0}, \psi_{h} \in U_{h}$. 
EM splitter: Given initial approximations $\boldsymbol{E}_{h}^{-\frac{3}{2}}, \boldsymbol{E}_{h}^{-\frac{1}{2}}, \boldsymbol{D}_{h}^{-\frac{3}{2}}, \boldsymbol{D}_{h}^{-\frac{1}{2}}, H_{h}^{0}$, for any $n \geq 0$, find $\boldsymbol{D}_{h}^{n+\frac{1}{2}} \in \boldsymbol{V}_{h}^{0}, \boldsymbol{E}_{h}^{n+\frac{1}{2}} \in \boldsymbol{V}_{h}^{0}, H_{h}^{n+1} \in U_{h}$ such that

$$
\begin{aligned}
& \left(\delta_{\tau} \boldsymbol{D}_{h}^{n+\frac{1}{2}}, v_{h}\right)=\left(H_{h}^{n}, \nabla \times v_{h}\right), \\
& \epsilon_{0}\left(\lambda_{s 2} \delta_{\tau}^{2} E_{h}^{n+\frac{1}{2}}, \phi_{h}\right)+\epsilon_{0}\left(\lambda_{s 2} \omega_{s e}^{2} \overline{\boldsymbol{E}}_{h}^{n+\frac{1}{2}}, \phi_{h}\right)=\left(M_{s a} \delta_{\tau}^{2} \boldsymbol{D}_{h}^{n+\frac{1}{2}}, \phi_{h}\right)+\left(M_{s b} \overline{\boldsymbol{D}}_{h}^{n+\frac{1}{2}}, \phi_{h}\right), \\
& \mu_{0}\left(\delta_{\tau} H_{h}^{n+1}, \psi_{h}\right)=-\left(\nabla \times \boldsymbol{E}_{h}^{n+\frac{1}{2}}, \psi_{h}\right),
\end{aligned}
$$

hold true for any $v_{h} \in V_{h}^{0}, \phi_{h} \in V_{h}^{0}, \psi_{h} \in U_{h}$.

To simulate the wave propagation in the TO devices, we surround the physical domain by a PML, which was originally introduced by Berenger [5] to reduce the EM wave propagation in unbounded domains to bounded domains. Here we adopt a 2D unsplit PML tested in our previous work [18]:

$$
\begin{aligned}
& \epsilon_{0} \frac{\partial \boldsymbol{E}}{\partial t}+\left(\begin{array}{cc}
\sigma_{y} & 0 \\
0 & \sigma_{x}
\end{array}\right) \boldsymbol{E}=\nabla \times H, \\
& \mu_{0} \frac{\partial H}{\partial t}+\left(\sigma_{m x}+\sigma_{m y}\right) H+\nabla \times \boldsymbol{E}+\sigma_{m x} \sigma_{m y} Q+\sigma_{m y} \frac{\partial P_{y}}{\partial x}-\sigma_{m x} \frac{\partial P_{x}}{\partial y}=0, \\
& \mu_{0} \frac{\partial \boldsymbol{P}}{\partial t}=\boldsymbol{E}, \quad \mu_{0} \frac{\partial Q}{\partial t}=H,
\end{aligned}
$$

where $\sigma_{i}, \sigma_{m, i}, i=x, y$, are the electric and magnetic conductivities in the $x$ and $y$ directions, respectively, and $\boldsymbol{P}=\left(P_{x}, P_{y}\right)^{\prime}$ and $Q$ are auxiliary variables.

To couple the PML equations with the schemes proposed above for solving the TO device equations, we design a similar leap-frog scheme to solve the PML equations as follows: Given proper initial approximations $\boldsymbol{E}_{h}^{-\frac{1}{2}}, \boldsymbol{P}_{h}^{0}, H_{h}^{0}$, for any $n \geq 0$, find $\boldsymbol{E}_{h}^{n+\frac{1}{2}} \in$ $\boldsymbol{V}_{h}^{0}, \boldsymbol{P}_{h}^{n+1} \in \boldsymbol{V}_{h}^{0}, H_{h}^{n+1}, Q_{h}^{n+\frac{1}{2}} \in U_{h}$ such that

$$
\begin{aligned}
& \epsilon_{0}\left(\delta_{\tau} \boldsymbol{E}_{h}^{n+\frac{1}{2}}, \phi_{h}\right)+\left(\left(\begin{array}{cc}
\sigma_{y} & 0 \\
0 & \sigma_{x}
\end{array}\right) \widehat{\boldsymbol{E}}_{h}^{n+\frac{1}{2}}, \phi_{h}\right)=\left(H_{h}^{n}, \nabla \times \phi_{h}\right), \\
& \mu_{0}\left(\delta_{\tau} \boldsymbol{P}_{h}^{n+1}, v_{h}\right)=\left(\boldsymbol{E}_{h}^{n+\frac{1}{2}}, v_{h}\right), \\
& \mu_{0}\left(\delta_{\tau} Q_{h}^{n+\frac{1}{2}}, u_{h}\right)=\left(H_{h}^{n}, u_{h}\right), \\
& \mu_{0}\left(\delta_{\tau} H_{h}^{n+1}, \psi_{h}\right)+\left(\left(\sigma_{m x}+\sigma_{m y}\right) \widehat{H}_{h}^{n+1}, \psi_{h}\right)+\left(\nabla \times \boldsymbol{E}_{h}^{n+\frac{1}{2}}, \psi_{h}\right) \\
& \quad+\left(\sigma_{m x} \sigma_{m y} Q_{h}^{n+\frac{1}{2}}, \psi_{h}\right)+\left(\sigma_{m y} \frac{\widehat{P}_{h, y}^{n+1}}{\partial x}, \psi_{h}\right)-\left(\sigma_{m x} \frac{\partial \widehat{P}_{h, x}^{n+1}}{\partial y}, \psi_{h}\right)=0,
\end{aligned}
$$

hold true for any $v_{h} \in V_{h}^{0}, \phi_{h} \in V_{h}^{0}, u_{h} \in U_{h}, \psi_{h} \in U_{h}$. 


\section{Numerical results}

In this section, we provide some numerical simulations showing the effectiveness of our time-domain mathematical models and the corresponding FETD schemes. In our simulations, we fix the physical domain $\Omega=[-0.8,0.8] m \times[-0.8,0.8] m$ discretized by an unstructured triangular meshes with mesh size $h=0.008$, and impose the plane wave source by the function $H_{z}=0.1 \sin (\omega t)$, where $\omega=2 \pi f$ and the source wave operating frequency $f=1.0 \mathrm{GHz}$. Furthermore, we surround the physical domain by a perfectly matched layer with 12 uniform rectangular cells in all directions.

Example 4.1. Simulation of EM concentrator. In this example, we consider a cylindrical electromagnetic concentrator with $a=0.1 \mathrm{~m}, b=0.3 \mathrm{~m}, c=0.4 \mathrm{~m}$. Our computational mesh contains 159638 total edges, 93696 total triangular elements, and 9360 total rectangular elements. We choose time step size $\tau=8 \times 10^{-13} s$, and the total number of time steps $N=15000$. To see how wave propagates in the concentrator device, we plot the $E_{y}$ fields at different times in Fig. 4, which show clearly how wave gets distorted in the metamaterial region. From Fig. 4, we can see that the structure has little scattering across the medium interfaces and it has the electromagnetic concentration effect.
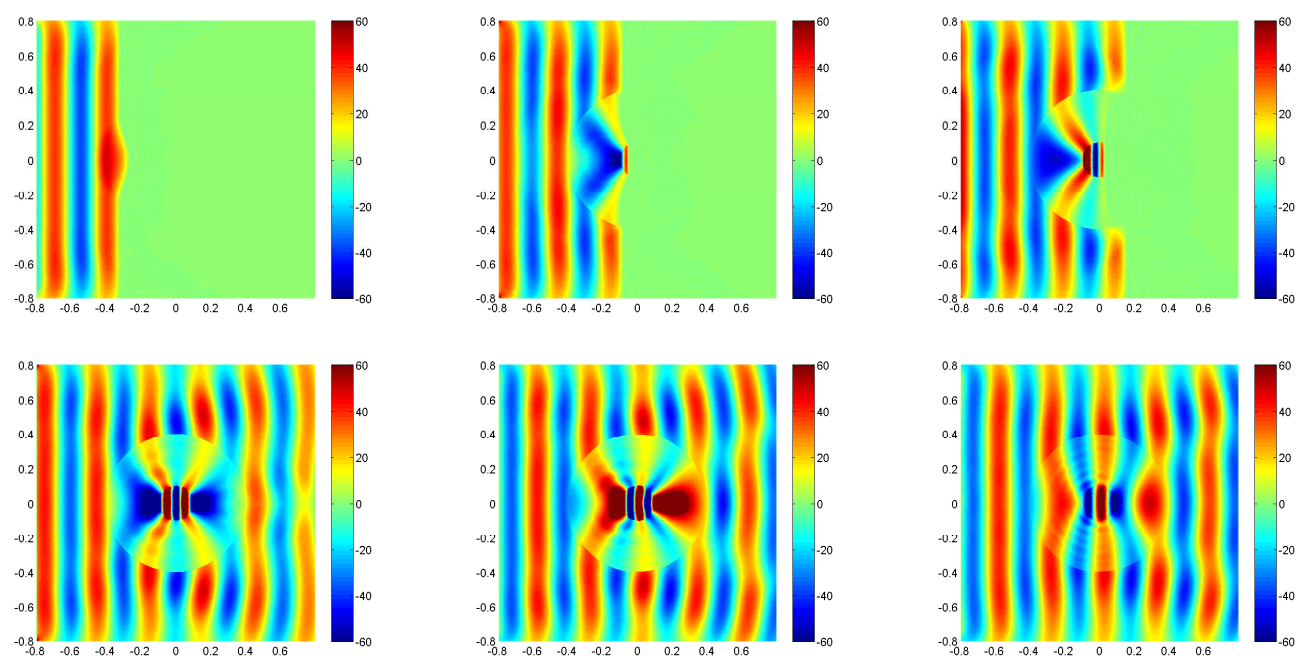

Figure 4: Snapshots of electric fields $E_{y}$ for the simulation of EM concentrator: Top left: 2000 time steps (top left); 3000 steps (top middle); 4000 steps (top right); 8000 steps (bottom left); 10000 steps (bottom middle); 15000 steps (bottom right).

Example 4.2. Simulation of EM rotator. In this example, we consider a time-domain cylindrical rotation cloak. We choose $R_{1}=0.2 m, R_{2}=0.4 m, \theta=\frac{\pi}{2}$, and our final mesh yields 159094 total edges, 92160 total triangular elements, and 10224 total rectangular elements. In our simulation, we choose the time step size $\tau=2.5 \times 10^{-13} \mathrm{~s}$, and the total number of 

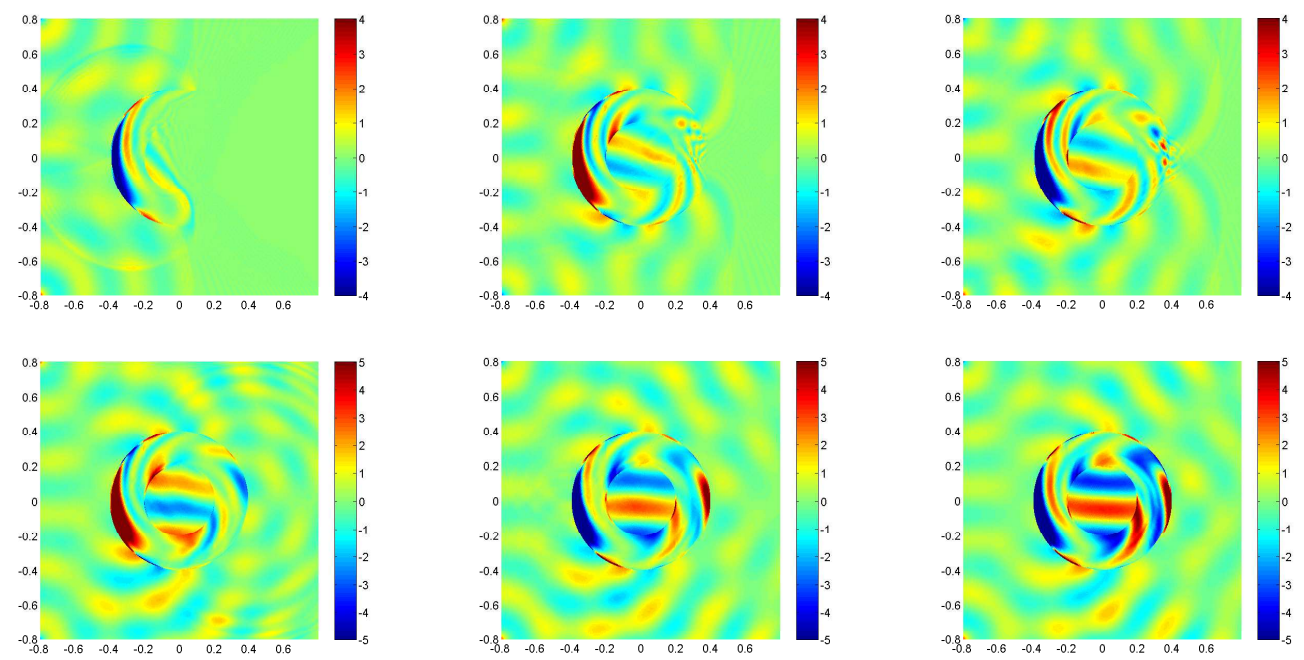

Figure 5: Snapshots of electric fields $E_{x}$ for the simulation of the EM rotator: 12000 steps (top left); 18000 steps (top middle); 20000 steps (top right); 30000 steps (bottom left); 40000 steps (bottom middle); 60000 steps (bottom right).
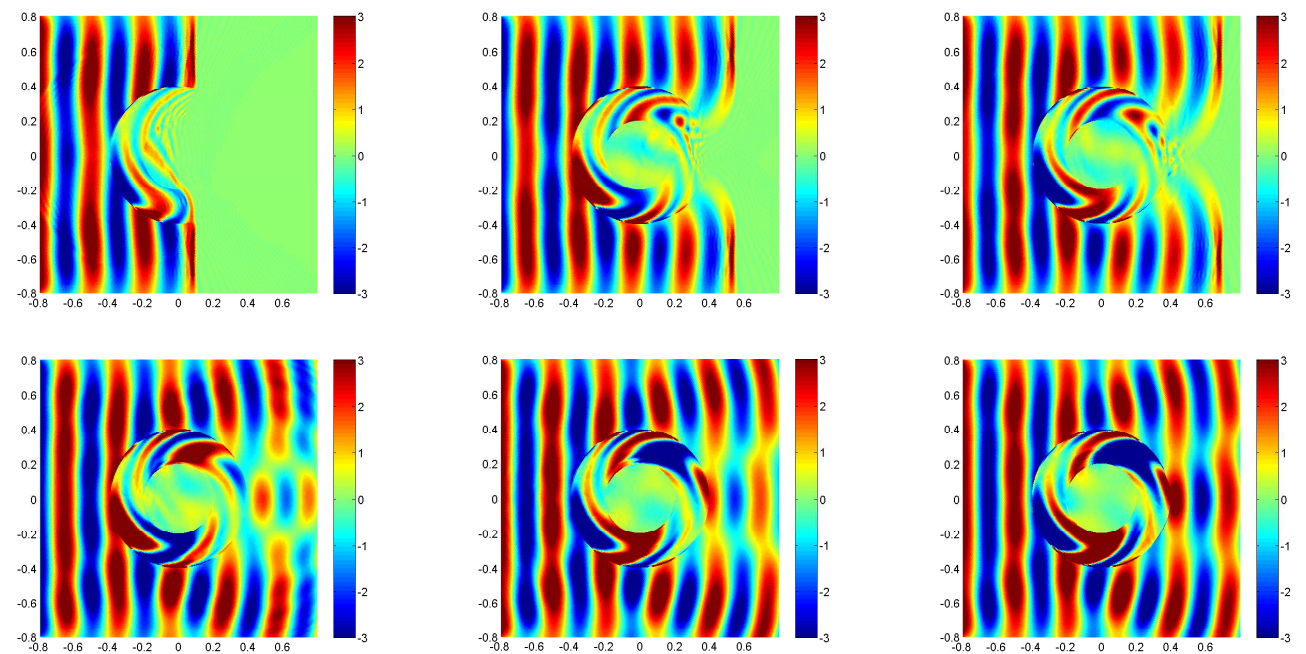

Figure 6: Snapshots of electric fields $E_{y}$ for the simulation of the EM rotator: 12000 steps (top left); 18000 steps (top middle); 20000 steps (top right); 30000 steps (bottom left); 40000 steps (bottom middle); 60000 steps (bottom right).

time steps $N=60000$, i.e., the final simulation time becomes $T=15$ nanosecond (ns). To see how wave propagates in the rotator, we plot the $E=\left(E_{x}, E_{y}\right)$ fields at different time steps in Figs. 5 and 6, which show clearly how wave gets distorted in the metamaterial region. From those pictures, we can see that the structure has very little scattering and it has the clearly rotation effect as obtained in [9, Fig. 4]. 
Example 4.3. Simulation of EM splitter. In this example, we consider a time-domain EM wave splitter. We choose the metamaterial region $\Omega=[-0.1,0.1] \times[-0.8,0.8]$, which is partitioned by a mesh with 160646 total edges, 92672 total triangular elements, and 10608 total rectangular elements. In our simulation, we choose the time step size $\tau=2 \times 10^{-13}$ $\mathrm{s}$, and the total number of time steps $N=40000$, i.e., the final simulation time $T=8 \mathrm{~ns}$.

First, we simulate the EM wave shifter with a parameter $k=2$. To see how wave propagates in this shifter structure, we plot the $E_{y}$ fields at different time steps in Fig. 7, which clearly show how wave gets shifted up in the metamaterial region. Fig. 7 shows clearly that the structure has very little scattering and shifts up the incoming EM wave.

Then we simulate the EM wave shifter with parameter $k=-2$. Snapshots of $E_{y}$ fields are plotted in Fig. 8, which clearly show how the incoming wave gets shifted down in the metamaterial region with little scattering.

Finally, we simulate the EM wave splitter. The moving parameter is chosen as $k=2$ in domain $\Omega_{2}=[-0.1,0.1] \times[0,0.8]$, and $k=-2$ in domain $\Omega_{3}=[-0.1,0.1] \times[-0.8,0]$. To see how wave propagates in the shifter structure, we plot the $E_{y}$ fields at different time steps in Fig. 9, which clearly show how wave gets shifted up and down in metamaterial regions with $k=2$ and $k=-2$, respectively.
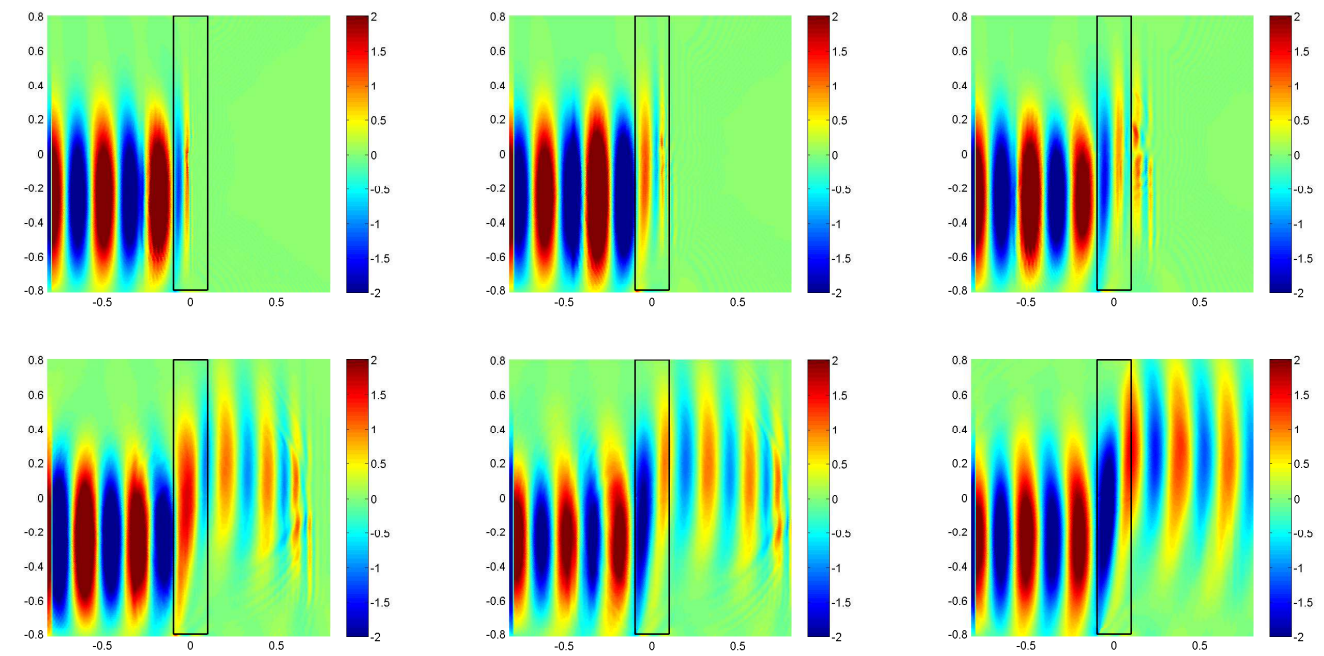

Figure 7: Snapshots of electric fields $E_{y}$ for the simulation of the EM wave shifter with parameter $k=2: 15000$ steps (top left); 18000 steps (top middle); 20000 steps (top right); 28000 steps (bottom left); 30000 steps (bottom middle); 40000 steps (bottom right).

\section{Conclusions}

In this paper, we propose the explicit FETD method for simulating EM transformation optics devices, including the EM concentrator, rotator and splitter. We first use trans- 

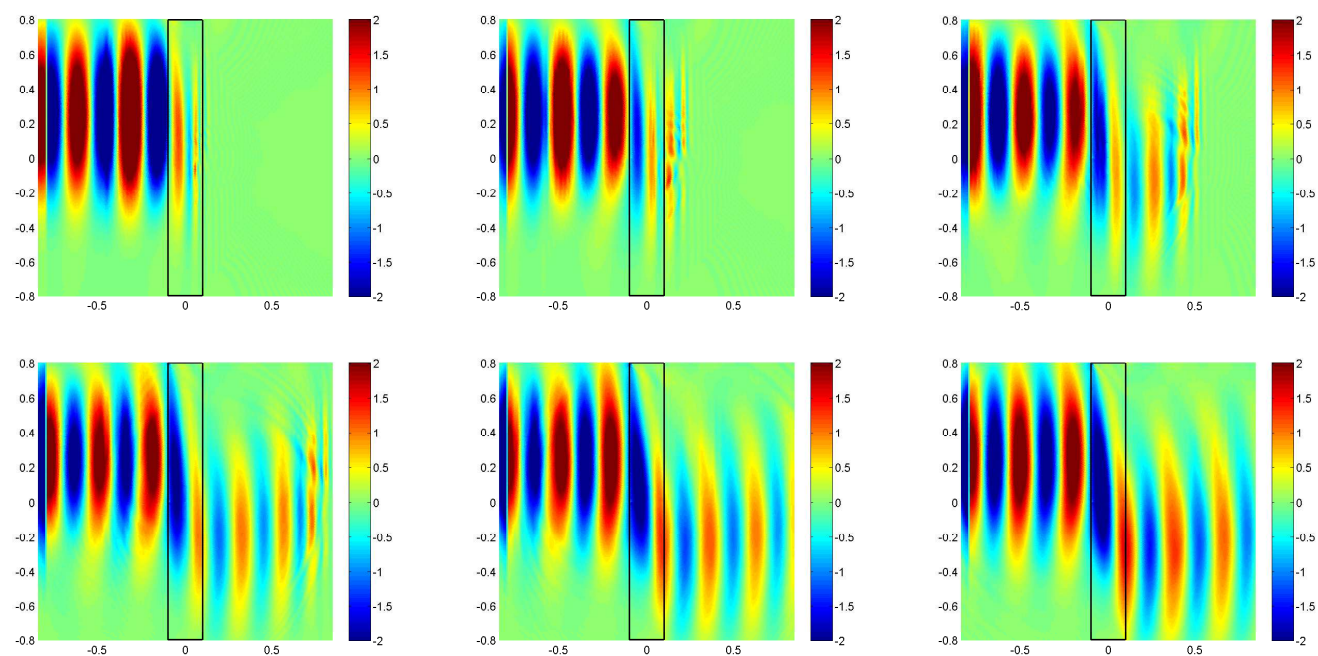

Figure 8: Snapshots of electric fields $E_{y}$ for the simulation of the EM wave shifter with parameter $k=2: 15000$ steps (top left); 18000 steps (top middle); 20000 steps (top right); 28000 steps (bottom left); 30000 steps (bottom middle); 40000 steps (bottom right).
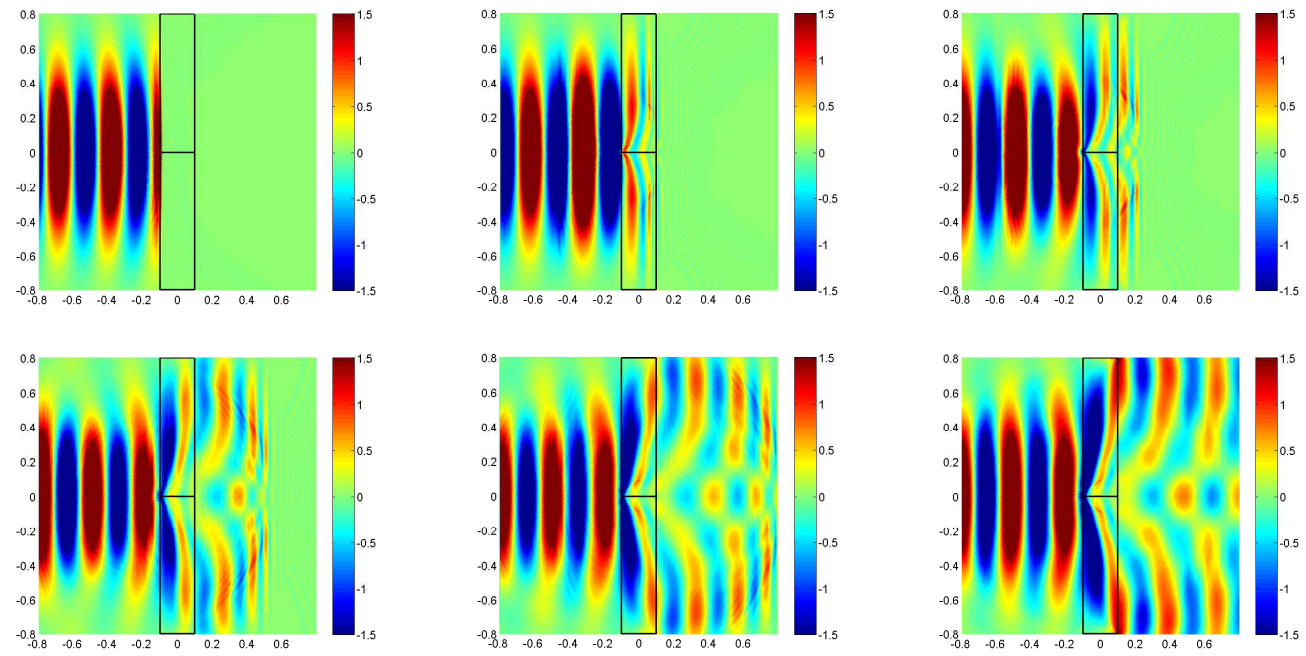

Figure 9: Snapshots of electric fields $E_{y}$ for the simulation of EM wave splitter: 12000 steps (top left); 18000 steps (top middle); 20000 steps (top right); 25000 steps (bottom left); 30000 steps (bottom middle); 40000 steps (bottom right).

formation optics to derive the device's permittivity and permeability, then establish the time-domain mathematical modeling equations, and finally we develop the fully discrete FETD method for solving these modeling equations. Numerical results are presented to demonstrate the effectiveness of our models and the corresponding FETD scheme. De- 
tailed mathematical analysis of these modeling equations will be investigated in our future work. More efficient numerical schemes will be explored in the future too.

\section{Acknowledgments}

The authors would like to thank the referees for their careful reading and kind suggestions for improving the paper, and this work was partially supported by NSFC Projects (11771371, 11671340), Hunan Education Department Projects (15B236, YB2015B027), Hunan NSF (2017jj3304), NSFC Key Projects (91430213, 91630205), NSF grant (DMS1416742), Guangdong Provincial Engineering Technology Research Center for Data Science.

\section{References}

[1] H. Ammari, H. Kang, H. Lee, M. Lim and S. Yu, Enhancement of near cloaking for the full Maxwell equations, SIAM J. Appl. Math. 73(6) (2013) 2055-2076.

[2] H. Banks, V. Bokil and N. Gibson, Analysis of stability and dispersion in a finite element method for Debye and Lorentz dispersive media, Numer. Methods Partial Differ. Equ. 25 (2009) 885-917.

[3] G. Bao, P. Li and H. Wu, An adaptive edge element method with perfectly matched absorbing layers for wave scattering by biperiodic structures, Math. Comput. 79 (2010) 1-34.

[4] R. Beck, R. Hiptmair, R.H.W. Hoppe and B. Wohlmuth, Residual based a posteriori error estimators for eddy current computation, Math. Model. Numer. Anal. 34 (2000) 159-182.

[5] J.P. Berenger, A perfectly matched layer for the absorption of electromagnetic waves, J. Comput. Phys. 114 (1994) 185-200.

[6] D. Boffi, P. Fernandez and I. Perugia, Computational models of electromagnetic resonators: analysis of edge element approximation, SIAM J. Numer. Anal. 36 (1999) 1264-1290.

[7] S.C. Brenner, J. Gedicke and L.-Y. Sung, Hodge decomposition for two-dimensional timeharmonic Maxwell's equations: impedance boundary condition, Math. Methods Appl. Sci. 40(2) (2017) 370-390.

[8] G. Castaldi, S. Savoia, V. Galdi, A. Alu and N. Engheta, PT metamaterials via complexcoordinate transformation optics, Phys. Rev. Lett. 110 (2013) 173901.

[9] H. Chen and C.T. Chen, Electromagnetic wave manipulation by layered systems using the transformation media concept, Phys. Rev. B 78 (2008) 054204.

[10] H. Chen, B.Hou, S.Chen, X. Ao, W. Wen and C.T. Chan, Design and experimental realization of a broadband transformation media field rotator at microwave frequencies, Phys. Rev. Lett. $102(18)(2009) 183903$.

[11] Z. Chen, Q. Du and J. Zou, Finite element methods with matching and nonmatching meshes for Maxwell equations with discontinuous coefficients, SIAM J. Numer. Anal. 37 (2000) 15421570 .

[12] L. Demkowicz, J. Kurtz, D. Pardo, M. Paszynski, W. Rachowicz and A. Zdunek, Computingwith hp finite elements. II. Frontiers: three-dimensional Elliptic and Maxwell problems with applications. Chapman Hall/CRC, Boca Raton, 2007.

[13] A. Greenleaf, Y. Kurylev, M. Lassas and G. Uhlmann, Cloaking devices, electromagnetic wormholes, and transformation optics, SIAM Rev. 51 (2009) 3-33. 
[14] F. Guevara-Vasquez, G.W. Milton and D. Onofrei, Broadband exterior cloaking, Opt. Express 17 (2009) 14800-14805.

[15] Y. Hao and R. Mittra, FDTD Modeling of Metamaterials: Theory and Applications, Artech House Publishers, 2008.

[16] J.S. Hesthaven and T. Warburton, Nodal high-order methods on unstructured grids: I. Timedomain solution of Maxwell's equations, J. Comput. Phys. 181 (2002) 186-221.

[17] R. Hiptmair, Finite elements in computational electromagnetism, Acta Numer. 11 (2002) 237339.

[18] Y. Huang, J. Li, and W. Yang, Modeling backward wave propagation in metamaterials by the finite element time-domain method, SIAM J. Sci. Comput. 35(1) (2013) B248-B274.

[19] Y. Huang, J. Li and W. Yang, Interior penalty DG methods for Maxwell's equations in dispersive media, J. Comput. Phys. 230(12) (2011) 4559-4570.

[20] W.X. Jiang, T.J. Cui, Q. Cheng, J.Y. Chin, X.M. Yang, R. Liu, and D.R. Smith, Design of arbitrarily shaped concentrators based on conformally optical transformation of nonuniform rational B-spline surfaces, Appl. Phys. Lett. 92 (2008) 264101.

[21] R. V. Kohn, H. Shen, M. S. Vogelius, and M. I. Weinstein, Cloaking via change of variables in electric impedance tomography, Inverse Problems 24(1) (2008) 015016.

[22] J. Li and Y. Huang, Time-Domain Finite Element Methods for Maxwell's Equations in Metamaterials, Springer Ser. Comput. Math. 43, Springer, New York, 2013.

[23] J. Li, Y. Huang and W. Yang, Well-posedness study and finite element simulation of timedomain cylindrical and elliptical cloaks, Math. Comp. 84 (2015) 543-562.

[24] J. Li, Y. Huang and W. Yang, Developing a time-domain finite-element method for modeling of electromagnetic cylindrical cloaks, J. Comput. Phys. 231 (2012) 2880-2891.

[25] J. Li, Y. Huang, W. Yang and A. Wood, Mathematical analysis and time domain finite element simulation of carpet cloak, SIAM J. Appl. Math. 74(4) (2014) 1136-1151.

[26] J. Li and J. B. Pendry, Hiding under the carpet: A new strategy for cloaking, Phys. Rev. Lett. $101(2008) 203901$.

[27] R. Liu, C. Ji, J.J. Mock, J.Y. Chin, T.J. Cui and D.R. Smith, Broadband ground-plane cloak, Science 323 (2009) 366-369.

[28] T. Lu, P. Zhang and W. Cai, Discontinuous Galerkin methods for dispersive and lossy Maxwell's equations and PML boundary conditions, J. Comput. Phys. 200 (2004) 549-580.

[29] P. Markos and C.M. Soukoulis, Wave Propagation: From Electrons to Photonic Crystals and Left-Handed Materials, Princeton University Press, 2008.

[30] P. Monk, Finite Element Methods for Maxwell's Equations, Oxford University Press, Oxford, 2003.

[31] E.E. Narimanov and A.V. Kildishev, Optical black hole: Broadband omnidirectional light absorber, Appl. Phys. Lett. 95 (2009) 041106.

[32] J.B. Pendry, D. Schurig and D.R. Smith, Controlling electromagnetic fields, Science 312 (2006) 1780-1782.

[33] M. Rahm, D. Schurig, D.A. Roberts, S.A. Cummer, D.R. Smith and J.B. Pendry, Design of electromagnetic cloaks and concentrators using form-invariant coordinate transformations of Maxwell's equations, Photonics and Nanostructures-fundamentals and Applications 6(1) (2008) 87-95.

[34] C. Scheid and S. Lanteri, Convergence of a Discontinuous Galerkin scheme for the mixed time domain Maxwells equations in dispersive media, IMA J. Numer. Anal. 33(2) (2013) 432-459.

[35] G. Shvets and I. Tsukerman, Plasmonics and Plasmonic Metamaterials: Analysis and Appli- 
cations, World Scientific, 2012.

[36] W. Wang, L. Lin, J. Ma, C. Wang, J. Cui, C. Du and X. Luo, Electromagnetic concentrators with reduced material parameters based on coordinate transformation, Optics Express 16 (2008) 11431-11437.

[37] D.H. Werner and D.H. Kwon (eds.), Transformation Electromagnetics and Metamaterials: Fundamental Principles and Applications, Springer, New York, 2013.

[38] Z. Xie, J. Wang, B. Wang and C. Chen, Solving Maxwell's equation in meta-materials by a CG-DG method, Commun. Comput. Phys. 19(5) (2016) 1242-1264.

[39] W. Yang, Y. Huang and J. Li, Developing a time-domain finite element method for the Lorentz metamaterial model and applications, J. Sci. Comput. 68(2) (2016) 438-463.

[40] W. Yang, J. Li and Y. Huang, Modeling and analysis of the optical black hole in metamaterials by the finite element time-domain method, Comput. Methods Appl. Mech. Engrg. 304 (2016) 501-520.

[41] Z. Yang, L.L. Wang, Z. Rong, B. Wang and B. Zhang, Seamless integration of global Dirichletto-Neumann boundary condition and spectral elements for transformation electromagnetics, Comput. Methods Appl. Mech. Engrg. 301 (2016) 137-163.

[42] Y. Zhao, C. Argyropoulos and Y. Hao, Full-wave finite-difference time-domain simulation of electromagnetic cloaking structures, Optics Express 16 (2008) 6717-6730.

[43] L. Zhong, L. Chen, S. Shu, G. Wittum and J. Xu, Convergence and optimality of adaptive edge finite element methods for time-harmonic Maxwell equations, Math. Comp. 81(278) (2012) 623-642,

[44] X. Zhu, B. Liang, W. Kan, X. Zou and J. Cheng, Acoustic cloaking by a superlens with singlenegative materials, Phys. Rev. Lett. 106 (2011) 014301. 